\title{
New Fast SCFT Algorithm Applied to Binary Diblock Copolymer/ Homopolymer Blends
}

\author{
M. W. Matsen ${ }^{\dagger}$ \\ Department of Physics, University of Reading, Whiteknights, Reading RG6 6AF, United Kingdom \\ Received J uly 4, 2003; Revised Manuscript Received October 6, 2003

\begin{abstract}
We present an efficient strategy for mapping out the classical phase behavior of block copolymer systems using self-consistent-fiel d theory. With our new algorithm, the complete sol ution of a classical block copolymer phase can be evaluated typically in a fraction of a second on a single-processor computer, even for highly segregated melts. This is accomplished by implementing the standard unitcell approximation for the cylindrical and spherical phases and solving the resulting equations using a Bessel function expansion. Here the method is used to investigate blends of AB diblock copolymer and A
\end{abstract} \\ homopolymer, concentrating on the situation where the two molecules are of similar size.
}

\section{Introduction}

The ability of block copolymers to combine chemically distinct polymeric materials and to do so in a variety of periodically ordered microstructures offers an impressive range of novel behaviors. ${ }^{1}$ However, the need to accurately synthesize complex molecules in order to target desired behavior remains a serious obstacle impeding their commercial exploitation. Fortunately, there is an al ternative to controlling material properties via synthesis, and that is the use of additives or, in other words, the blending of two or more molecular species. Not only is this a trivial way of adjusting the properties, it can also produce new behaviors not present in neat (i.e., pure) block copolymer materials.

Unfortunately, blending introduces a new problem, that of a daunting large parameter space. In addition to a variety of molecular species to contend with, there is also the blend composition to consider. Even a simple binary blend of $A B$ diblock copolymer and A-type homopolymer involves four relevant parameters: the degree of segregation, the relative molecular weights, the composition of the diblock, and the blend composition. As more distinct molecules are added to the mix or as the molecules become more complex, the number of parameters rapidly expands. Exploring the phase behavior of such vast parameter spaces by experiments al one is a mammoth challenge, and without theoretical guidance there would be little chance of developing a thorough understanding of the overall behavior.

As fortune would have it, there is good evidence that self-consistent-field theory (SCFT) $)^{2,3}$ can fill this particular need. This state-of-the-art theory has already developed an unrivaled track record for accurately predicting the phase behavior of a wide range of systems, such as neat $A B$ diblock copolymer melts, ${ }^{4}$ neat ABC triblock copolymer melts, ${ }^{5}$ and binary diblock/ diblock blends. ${ }^{6}$ When implemented fully, SCFT provides exact mean-field predictions, whereas in general the alternative methods ${ }^{7-11}$ only provide approximations to mean-field theory. The main drawback of SCFT is that it can be computationally demanding as either the segregation or the periodicity of a morphology increases. Even still, it is far less computational than either Monte

\footnotetext{
† E-mail: m.w.matsen@reading.ac.uk.
}

Carlo12,13 or molecular dynamics ${ }^{14}$ simulations, for which accurately locating just a single point on a phase boundary remains a nontrivial task. ${ }^{12}$

We have previously15,16 applied SCFT to binary diblock/homopolymer blends, producing results in good qualitative agreement with existing experiments. However, the numerical demands of SCFT coupled with the complexity of the phase diagram restricted our study to relatively weak segregations of $\chi \mathrm{N} \leq 12$, where $\chi$ is the usual Flory-Huggins parameter and $\mathrm{N}$ is the degree of polymerization of the diblock. J anert and Schick ${ }^{17}$ extended these calculations to stronger segregations, $\chi \mathrm{N}$ $\lesssim 18$, by disregarding the complex morphologies (e.g., gyroid, double-diamond, and perforated-lamellar) and focusing only on the classical phases (e.g., Iamellar, cylindrical, and spherical). Even without the complex phases, their partial (or classical) phase diagrams provide the essential behavior of these blends, since it is well understood that the complex phases generally exist in narrow channels between the lamellar and cylindrical phases due to their intermediate interfacial curvature. ${ }^{4,18}$

Here we go one step further than J anert and Schick and approximate the Wigner-Seitz unit cells of the cylindrical and spherical phases by circular and spherical unit cells, respectively. This is the unit-cell approximation (UCA), the use of which was normal practice in earlier SCFT calculations 2,19 and remains so in alternative theories. ${ }^{7,8}$ The UCA has been demonstrated to be a good approximation in neat diblock copolymer melts ${ }^{20}$ and presumably will remain so for most block copolymer blends. Whereas previous UCAbased SCFT calculations solved the self-consistent-field equations in real space, 2,19 we will instead use Bessel function expansions. Not only does this offer significant advantages in controlling numerical accuracy, it also provides an exceptionally efficient algorithm. In fact, it becomes feasible for a single-processor computer to solve the self-consistent-field equations for any given classical phase, optimize its periodicity, and evaluate its free energy to an accuracy of 1 part in $10^{9}$, all in fraction of a second. This is for experimentally rel evant segregations of $\chi \mathrm{N} \sim 20$, but even for exceptionally high segregations of $\chi \mathrm{N} \sim 100$, the computational time generally remains well under a minute. 


\section{Theory}

This section discusses our new SCFT procedure in the context of binary diblock copolymer/homopolymer blends. The theory is based on the same standard Gaussian chain model used by virtually all block copolymer calculations. ${ }^{4,9}$ The A and B polymer segments are assumed to be completely flexible and incompressible, each with a statistical length of a and a fixed volume of $1 / \rho_{0}$, and their mutual interaction is controlled by the usual Flory-Huggins $\chi$ parameter. The $A$ and $B$ blocks of each copolymer are composed of $\mathrm{fN}$ and $(1-\mathrm{f}) \mathrm{N}$ segments, respectively, while each homopolymer consists of $\alpha \mathrm{N}$ A-type segments.

The SCFT for a blend can be performed in either the canonical formalism devel oped by $\mathrm{H}$ ong and $\mathrm{NoOlandi}{ }^{21}$ or, equivalently, the grand-canonical approach previously introduced by us. ${ }^{15}$ In the former ensemble, the volume fraction of homopolymer, $\phi$, is fixed, whereas in the latter, the quantity of homopolymer is controlled by a chemical potential, $\mu$. Both ensembles have their distinct advantages, and therefore we use a combination of the two. For detailed derivations, we refer the reader to the original papers. ${ }^{15,21}$ Here we just present the key equations for each approach in a unified notation in order to facilitate their combined use and to provide an ample basis for discussing our computational method.

A. SCFT for Ordered Phases. Because of the unitcell approximation (UCA), our algorithm is restricted to the three classical morphologies: lamellae (L), cylinders (C), and spheres (S). The UCA is used to si mplify the calculation for $\mathrm{C}$ and $\mathrm{S}$ by approximating their Wigner-Seitz unit cells by a circular cylinder and a sphere, respectively. ${ }^{2}$ With the resulting rotational symmetry, all quantities will depend only on the distance, $r$, from the center of the unit cell. For convenience, this is expressed in terms of the dimensionless coordinate, $\rho \equiv \mathrm{r} / \mathrm{R}$, where $\mathrm{R}$ is the radius of the unit cell. In the case of $L$, which is still treated exactly, $r$ measures the distance from the center of a lamellar domain, and R corresponds to half the lamellar period.

The basic idea behind SCFT is that the molecular interactions experienced by $A$ and $B$ segments are approximated by the static fields

$$
\begin{gathered}
\mathrm{w}_{\mathrm{A}}(\rho)=\chi \mathrm{N} \phi_{\mathrm{CB}}(\rho)+\xi(\rho) \\
\mathrm{w}_{\mathrm{B}}(\rho)=\chi \mathrm{N}\left[\phi_{\mathrm{CA}}(\rho)+\phi_{\mathrm{h}}(\rho)\right]+\xi(\rho)
\end{gathered}
$$

where $\phi_{C A}(\rho)$ is the dimensionless concentration of the copolymer A blocks, $\phi_{\mathrm{CB}}(\rho)$ is the concentration of B blocks, $\phi_{\mathrm{h}}(\rho)$ is the homopolymer distribution, and $\xi(\rho)$ is a Lagrange multiplier field used to enforce the incompressibility constraint

$$
\phi_{\mathrm{CA}}(\rho)+\phi_{\mathrm{CB}}(\rho)+\phi_{\mathrm{h}}(\rho)=1
$$

With this mean-field approximation, the statistical mechanics of the problem reduces to that of noninteracting molecules. For a diblock copolymer, this requires the evaluation of two partial partition functions corresponding to a molecule with its sNth segment (as measured from its $A$ end) fixed at a distance $r=\rho R$ from the center of the unit cell. The partial partition function, $q_{c}(\rho, s)$, for the first $s \mathrm{~N}$ segments satisfies

$$
\frac{\partial}{\partial s} q_{c}(\rho, s)=\left\{\begin{array}{l}
\frac{a^{2} N}{6 R^{2}} \nabla^{2} q_{c}(\rho, s)-w_{A}(\rho) q_{c}(\rho, s), \text { if } s<f \\
\frac{a^{2} N}{6 R^{2}} \nabla^{2} q_{c}(\rho, s)-w_{B}(\rho) q_{c}(\rho, s), \text { if } s>f
\end{array}\right.
$$

with the initial condition $\mathrm{q}_{\mathrm{c}}(\rho, 0)=1$. For our particular symmetries, the Laplacian is given by

$$
\nabla^{2} \equiv \frac{1}{\rho^{m}} \frac{\partial}{\partial \rho} \rho^{m} \frac{\partial}{\partial \rho}
$$

where the integer $\mathrm{m}$ takes on the value 0,1 , or 2 depending on whether the morphology is $L, C$, or $S$, respectively. The other partial partition function, $\mathrm{q}_{c}^{\dagger}(\rho, \mathrm{s})$, for the remaining $(1-\mathrm{s}) \mathrm{N}$ segments satisfies the same diffusion equation (4), but with the right-hand side multiplied by -1 , and obeys the condition $\mathrm{q}_{c}^{\dagger}(\rho, 1)$ $=1$. The homopolymer only requires one partial partition function, $\mathrm{q}_{\mathrm{h}}(\rho, \mathrm{s})$, corresponding to a chain of $\mathrm{sN}$ segments with one end fixed at $r=\rho R$. It satisfies

$$
\frac{\partial}{\partial s} q_{h}(\rho, s)=\frac{a^{2} N}{6 R^{2}} \nabla^{2} q_{h}(\rho, s)-w_{A}(\rho) q_{h}(\rho, s)
$$

and obeys $\mathrm{q}_{\mathrm{h}}(\rho, 0)=1$. In terms of these functions, the full partition function for a copolymer molecule acted upon by the mean fields is

$$
\mathcal{L}_{\mathrm{c}}=\mathrm{V}(\mathrm{m}+1) \int_{0}^{1} \rho^{\mathrm{m}} \mathrm{q}_{\mathrm{c}}(\rho, \mathrm{s}) \mathrm{q}_{\mathrm{c}}^{\dagger}(\rho, \mathrm{s}) \mathrm{d} \rho
$$

and that for a homopolymer is

$$
\mathcal{L}_{\mathrm{h}}=\mathrm{V}(\mathrm{m}+1) \int_{0}^{\alpha} \rho^{\mathrm{m}} \mathrm{q}_{\mathrm{h}}(\rho, \mathrm{s}) \mathrm{q}_{\mathrm{h}}(\rho, \alpha-\mathrm{s}) \mathrm{d} \rho
$$

where $\mathrm{V}$ is the total volume of the blend.

Once the partition functions are evaluated, the segment concentrations are obtained by simple integration. In the canonical ensemble, they are given by

$$
\begin{aligned}
\phi_{\mathrm{CA}}(\rho) & =\frac{(1-\phi) \mathrm{V}}{\mathcal{2}_{\mathrm{c}}} \int_{0}^{\mathrm{f}} \mathrm{q}_{\mathrm{c}}(\rho, \mathrm{s}) \mathrm{q}_{\mathrm{c}}^{\dagger}(\rho, \mathrm{s}) \mathrm{ds} \\
\phi_{\mathrm{cB}}(\rho) & =\frac{(1-\phi) \mathrm{V}}{\mathcal{2}_{\mathrm{c}}} \int_{\mathrm{f}}^{1} \mathrm{q}_{\mathrm{c}}(\rho, \mathrm{s}) \mathrm{q}_{\mathrm{c}}^{\dagger}(\rho, \mathrm{s}) \mathrm{ds} \\
\phi_{\mathrm{h}}(\rho) & =\frac{\phi \mathrm{V}}{\alpha \mathcal{2}_{\mathrm{h}}} \int_{0}^{\alpha} \mathrm{q}_{\mathrm{h}}(\rho, \mathrm{s}) \mathrm{q}_{\mathrm{h}}(\rho, \alpha-\mathrm{s}) \mathrm{ds}
\end{aligned}
$$

and in the grand-canonical ensemble, they are

$$
\begin{gathered}
\phi_{\mathrm{CA}}(\rho)=\int_{0}^{f} q_{\mathrm{c}}(\rho, s) q_{c}^{\dagger}(\rho, s) d s \\
\phi_{\mathrm{CB}}(\rho)=\int_{\mathrm{f}}^{1} \mathrm{q}_{\mathrm{c}}(\rho, \mathrm{s}) \mathrm{q}_{\mathrm{c}}^{\dagger}(\rho, \mathrm{s}) \mathrm{ds} \\
\phi_{\mathrm{h}}(\rho)=\mathrm{z} \int_{0}^{\alpha} \mathrm{q}_{\mathrm{h}}(\rho, \mathrm{s}) \mathrm{q}_{\mathrm{h}}(\rho, \alpha-\mathrm{s}) \mathrm{ds}
\end{gathered}
$$

where $z \equiv \exp \left(\mu / \mathrm{k}_{\mathrm{B}} \mathrm{T}\right)$. With these expressions, it is now possible to adjust the fields, $\mathrm{w}_{\mathrm{A}}(\rho)$ and $\mathrm{w}_{\mathrm{B}}(\rho)$, so as to obtain a self-consistent solution to eqs $1-3$. In the canonical ensemble, an added constant to the fields has no effect on the statistical mechanics, and consequently 
the average value of $\xi(\rho)$ must be fixed; for simplicity, we set $\int \rho^{\mathrm{m}} \xi(\rho) \mathrm{d} \rho=0$.

To determine the preferred domain size, $R$, of each morphology and then to identify the equilibrium phase requires us to evaluate the free energies corresponding to the various self-consistent solutions. In the canonical ensemble, the free energy, $F_{c}$, is given by

$$
\begin{array}{r}
\frac{N F_{\mathrm{c}}}{\mathrm{k}_{\mathrm{B}} \mathrm{T} \rho_{0} \mathrm{~V}}=(1-\phi)\left[\ln \left(\frac{(1-\phi) \mathrm{V}}{\mathcal{Q}_{\mathrm{c}}}\right)-1\right]+ \\
\frac{\phi}{\alpha}\left[\ln \left(\frac{\phi \mathrm{V}}{\alpha \mathcal{L}_{\mathrm{h}}}\right)-1\right]-(\mathrm{m}+1) \chi \mathrm{N} \int_{0}^{1} \rho^{\mathrm{m}_{\left\{\phi_{\mathrm{CA}}\right.}(\rho)+} \\
\left.\phi_{\mathrm{h}}(\rho)\right\} \phi_{\mathrm{CB}}(\rho) \mathrm{d} \rho
\end{array}
$$

and in the grand-canonical ensemble, the free energy, $\mathrm{F}_{\mathrm{gc}}$ is

$$
\begin{array}{r}
\frac{N F_{g c}}{k_{B} T \rho_{0} V}=-\frac{\mathscr{Q}_{c}}{V}-\frac{z \mathscr{Q}_{h}}{V}-(m+1) \int_{0}^{1} \rho^{m}\left\{\chi N \left[\phi_{c A}(\rho)+\right.\right. \\
\left.\left.\phi_{\mathrm{h}}(\rho)\right\} \phi_{\mathrm{cB}}(\rho)+\xi(\rho)\right] \mathrm{d} \rho
\end{array}
$$

Since additive constants to the free energy have no real consequence, these expressions differ somewhat from paper to paper; for example, the -1 in the two square brackets of eq 15 is often omitted. We choose these particular expressions because they satisfy the relation $\mathrm{F}_{\mathrm{gc}}=\mathrm{F}_{\mathrm{c}}-\mu \mathrm{n}_{\mathrm{h}}$, where $\mathrm{n}_{\mathrm{h}} \equiv \phi \mathrm{V} \rho_{0} / \alpha \mathrm{N}$ is the number of homopolymer molecules in the blend.

B. Functional Expansion. Previous applications $s^{2,19,20}$ of the UCA have solved the self-consistent-field equations in real space, but we use what appears to be a far more efficient approach. This involves expanding all spatially dependent quantities, $\mathrm{g}(\rho)$, in terms of basis functions, $f_{i}(\rho)$, satisfying the symmetry of the phase under consideration. ${ }^{22}$ More specifically

$$
\mathrm{g}(\rho)=\sum_{\mathrm{i}=1}^{\infty} \mathrm{g}_{\mathrm{i}} \mathrm{f}_{\mathrm{i}}(\rho)
$$

where the first basis function is $f_{1}(\rho)=1$, with the remaining ones given by

$$
\mathrm{f}_{\mathrm{i}}(\rho)=\left\{\begin{array}{l}
\sqrt{2} \cos (\pi(\mathrm{i}-1) \rho), \text { for } \mathrm{L} \\
\mathrm{J}_{0}\left(\alpha_{\mathrm{i}} \rho\right) / / \mathrm{J}_{0}\left(\alpha_{\mathrm{i}}\right) \mid, \text { for } \mathrm{C} \\
\sqrt{\frac{2}{3}} \mathrm{j}_{0}\left(\beta_{\mathrm{i}} \rho\right) /\left|\mathrm{j}_{0}\left(\beta_{\mathrm{i}}\right)\right|, \text { for } \mathrm{S}
\end{array}\right.
$$

The coefficients, $\alpha_{\mathrm{i}}$ and $\beta_{\mathrm{i}}$, are the points in increasing order, where the Bessel function, $\mathrm{J}_{0}(\mathrm{x})$, and the spherical Bessel function, $\mathrm{j}_{0}(\mathrm{x}) \equiv \sin (\mathrm{x}) / \mathrm{x}$, have zero slope. For large $\mathrm{i}$, these coefficients can be approximated by ${ }^{23}$

$$
\begin{aligned}
& \alpha_{\mathrm{i}} \approx \frac{(4 \mathrm{i}-3) \pi}{4}-\frac{3}{2(4 \mathrm{i}-3) \pi}+\frac{3}{2(4 \mathrm{i}-3)^{3} \pi^{3}} \\
& \beta_{\mathrm{i}} \approx \frac{(2 \mathrm{i}-1) \pi}{2}-\frac{2}{(2 \mathrm{i}-1) \pi}-\frac{16}{3(2 \mathrm{i}-1)^{3} \pi^{3}}
\end{aligned}
$$

Each group of basis functions forms an orthonormal set satisfying

$$
(m+1) \int_{0}^{1} \rho^{m} f_{i}(\rho) f_{j}(\rho) d \rho=\delta_{i j}
$$

and they are eigenfunctions of the Laplacian operator

$$
\nabla^{2} \mathrm{f}_{\mathrm{i}}(\rho)=-\lambda_{\mathrm{i}} \mathrm{f}_{\mathrm{i}}(\rho)
$$

with eigenvalues given by

$$
\lambda_{\mathrm{i}}=\left\{\begin{array}{l}
\pi^{2}(\mathrm{i}-1)^{2}, \text { for } \mathrm{L} \\
\alpha_{\mathrm{i}}{ }^{2}, \text { for } \mathrm{C} \\
\beta_{\mathrm{i}}{ }^{2}, \text { for } \mathrm{S}
\end{array}\right.
$$

Using the orthogonality condition, eq 21 , it fol lows that coefficients in the expansion of $g(\rho)$ are given by

$$
g_{i}=(m+1) \int_{0}^{1} \rho^{m} g(\rho) f_{i}(\rho) d \rho
$$

Of course, it is impossible, in practice, to include all the coefficients, and thus the series in eq 17 must be truncated at $\mathrm{i}=\mathrm{M}$. Nevertheless, $\mathrm{M}$ can be chosen sufficiently large such that the resulting numerical inaccuracy is irrel evant.

In terms of the expansion, the coefficients of $\mathrm{q}_{\mathrm{c}}(\rho, \mathrm{s})$ satisfy a simple set of first-order linear ordinary differential equations

$$
\frac{\mathrm{d}}{\mathrm{ds}} \mathrm{q}_{\mathrm{c}, \mathrm{i}}(\mathrm{s})=\left\{\begin{array}{l}
\Sigma_{j} \mathrm{~A}_{\mathrm{ij}} \mathrm{q}_{\mathrm{c}, \mathrm{j}}(\mathrm{s}), \text { if } \mathrm{s}<\mathrm{f} \\
\Sigma_{j} \mathrm{~B}_{\mathrm{ij}} \mathrm{q}_{\mathrm{c}, \mathrm{j}}(\mathrm{s}), \text { if } \mathrm{s}>\mathrm{f}
\end{array}\right.
$$

The matrix elements of $\mathbf{A}$ are given by

$$
A_{i j} \equiv-\frac{a^{2} N}{6 R^{2}} \lambda_{i} \delta_{i j}-\sum_{k} w_{A, k} \Gamma_{i j k}
$$

where

$$
\Gamma_{\mathrm{ijk}} \equiv(\mathrm{m}+1) \int_{0}^{1} \rho^{m} \mathrm{f}_{\mathrm{i}}(\rho) \mathrm{f}_{\mathrm{j}}(\rho) \mathrm{f}_{\mathrm{k}}(\rho) \mathrm{d} \rho
$$

and the elements of $\mathbf{B}$ are given by an equivalent expression with $\mathrm{w}_{\mathrm{A}, \mathrm{k}}$ replaced by $\mathrm{w}_{\mathrm{B}, \mathrm{k}}$. The solution to eq 25 can be expressed as

$$
q_{c, i}(s)=\left\{\begin{array}{l}
T_{A, i 1}(s), \text { if } s \leq f \\
\sum_{j} T_{B, i j}(s-f) q_{c, j}(f), \text { if } s>f
\end{array}\right.
$$

where $\mathbf{T}_{\mathrm{A}}(\mathrm{s}) \equiv \exp (\mathrm{s} \mathbf{A})$ and $\mathbf{T}_{\mathrm{B}}(\mathrm{s}) \equiv \exp (\mathrm{sB})$ are referred to as transfer matrices. Similarly, the solution corresponding to $\mathrm{q}_{c}^{\dagger}(\rho, \mathrm{s})$ is

$$
q_{c, i}^{\dagger}(s)=\left\{\begin{array}{l}
\sum_{j} T_{A, i j}(f-s) q_{c, j}^{\dagger}(f), \text { if } s<f \\
T_{B, i 1}(1-s), \text { if } s \geq f
\end{array}\right.
$$

and that for $\mathrm{q}_{\mathrm{h}}(\rho, \mathrm{s})$ is simply

$$
\mathrm{q}_{\mathrm{h}, \mathrm{i}}(\mathrm{s})=\mathrm{T}_{\mathrm{A}, \mathrm{i}}(\mathrm{s})
$$

In practice, the transfer matrix, $\mathbf{T}_{\mathrm{A}}(\mathrm{s})$, is evaluated by first diagonalizing the real symmetric matrix, $\mathbf{A}=$ $\mathbf{V}_{A} \mathbf{D}_{A} \mathbf{V}_{A}^{\top}$. Once that is done, the elements of the transfer matrix are given by

$$
\mathrm{T}_{\mathrm{A}, \mathrm{ij}}(\mathrm{s})=\sum_{\mathrm{k}} \exp \left(\mathrm{sd}_{\mathrm{A}, \mathrm{k}}\right) \mathrm{V}_{\mathrm{A}, \mathrm{ik}} \mathrm{V}_{\mathrm{A}, \mathrm{jk}}
$$


where $d_{A, k} \equiv D_{A, k k}$ is the $k$ th eigenvalue of $\mathbf{A}$ and $\left(V_{A, 1 k}\right.$, $\left.V_{A, 2 k}, V_{A, 3}, \ldots\right)$ is the corresponding normalized eigenvector. Fortunately, there exists a very efficient and accurate procedure for diagonalizing real symmetric matrices, where the Householder method is used to find a similarity transformation to a tridiagonal matrix to which the so-called QL algorithm is applied. ${ }^{24}$

Once the eigenvalues and eigenvectors of $\mathbf{A}$ and $\mathbf{B}$ have been evaluated, we then compute the matrices

$$
\begin{aligned}
& \mathrm{I}_{\mathrm{cA}, \mathrm{jk}} \equiv \int_{0}^{\mathrm{f}} \mathrm{q}_{\mathrm{c}, \mathrm{j}}(\mathrm{s}) \mathrm{q}_{\mathrm{c}, \mathrm{k}}^{\dagger}(\mathrm{s}) \mathrm{ds} \\
& =\sum_{m, n}\left[\frac{\exp \left(f d_{A, m}\right)-\exp \left(f d_{A, n}\right)}{d_{A, m}-d_{A, n}}\right] \times \\
& \mathrm{V}_{\mathrm{A}, \mathrm{jm}} \mathrm{V}_{\mathrm{A}, \mathrm{kn}} \overline{\mathrm{q}}_{\mathrm{CA}, \mathrm{m}}(0) \overline{\mathrm{q}}_{\mathrm{CA}, \mathrm{n}}^{\dagger}(\mathrm{f}) \\
& \mathrm{I}_{\mathrm{cB}, j \mathrm{k}} \equiv \int_{\mathrm{f}}^{1} \mathrm{q}_{\mathrm{c}, \mathrm{j}}(\mathrm{s}) \mathrm{q}_{\mathrm{c}, \mathrm{k}}^{\dagger}(\mathrm{s}) \mathrm{ds} \\
& =\sum_{m, n}\left[\frac{\exp \left((1-f) d_{B, m}\right)-\exp \left((1-f) d_{B, n}\right)}{d_{B, m}-d_{B, n}}\right] \times \\
& \mathrm{V}_{\mathrm{B}, \mathrm{jm}} \mathrm{V}_{\mathrm{B}, \mathrm{kn}} \overline{\mathrm{q}}_{\mathrm{CB}, \mathrm{m}}(\mathrm{f}) \overline{\mathrm{q}}_{\mathrm{CB}, \mathrm{n}}^{\dagger}(1) \\
& \mathrm{I}_{\mathrm{h}, \mathrm{jk}} \equiv \int_{0}^{\alpha} \mathrm{q}_{\mathrm{h}, \mathrm{j}}(\mathrm{s}) \mathrm{q}_{\mathrm{h}, \mathrm{k}}(\alpha-\mathrm{s}) \mathrm{ds} \\
& =\sum_{m, n}\left[\frac{\exp \left(\alpha d_{A, m}\right)-\exp \left(\alpha d_{A, n}\right)}{d_{A, m}-d_{A, n}}\right] \times \\
& \mathrm{V}_{\mathrm{A}, \mathrm{m}} \mathrm{V}_{\mathrm{A}, \mathrm{kn}} \overline{\mathrm{q}}_{\mathrm{hA}, \mathrm{m}}(0) \overline{\mathrm{q}}_{\mathrm{hA}, \mathrm{n}}(0)
\end{aligned}
$$

where we have simplified the expressions by defining $\overline{\mathrm{X}}_{\mathrm{A}, \mathrm{i}} \equiv \sum_{\mathrm{j}} \mathrm{X}_{\mathrm{j}} \mathrm{V}_{\mathrm{A}, \mathrm{ji}}$ and $\mathrm{X}_{\mathrm{B}, \mathrm{i}} \equiv \sum_{\mathrm{j}} \mathrm{X}_{\mathrm{j}} \mathrm{V}_{\mathrm{B}, \mathrm{j}}$. Note that when the pair of eigenvalues in the denominators of eqs 33, 35, and 37 become equal, the quantities in square parentheses reduce to $f \exp \left(f d_{A, m}\right),(1-f) \exp \left((1-f) d_{B, m}\right)$, and $\alpha \exp \left(\alpha \mathrm{d}_{\mathrm{A}, \mathrm{m}}\right)$, respectively. Given thethree matrices, the segment concentrations are obtained from

$$
\begin{aligned}
\phi_{\mathrm{CA}, \mathrm{i}} & =\frac{1-\phi}{\mathrm{q}_{\mathrm{c}, 1}(1)} \sum_{\mathrm{j}, \mathrm{k}} \mathrm{I}_{\mathrm{cA}, \mathrm{jk}} \Gamma_{\mathrm{ijk}} \\
\phi_{\mathrm{cB}, \mathrm{i}} & =\frac{1-\phi}{\mathrm{q}_{\mathrm{c}, 1}(1)} \sum_{\mathrm{j}, \mathrm{k}} \mathrm{I}_{\mathrm{cB}, \mathrm{jk}} \Gamma_{\mathrm{ijk}} \\
\phi_{\mathrm{h}, \mathrm{i}} & =\frac{\phi}{\alpha \mathrm{q}_{\mathrm{h}, 1}(\alpha)} \sum_{\mathrm{j}, \mathrm{k}} \mathrm{I}_{\mathrm{h}, \mathrm{jk}} \Gamma_{\mathrm{ijk}}
\end{aligned}
$$

in the canonical ensemble and from

$$
\begin{aligned}
& \phi_{\mathrm{cA}, \mathrm{i}}=\sum_{\mathrm{j}, \mathrm{k}} \mathrm{I}_{\mathrm{cA}, \mathrm{jk}} \Gamma_{\mathrm{ijk}} \\
& \phi_{\mathrm{cB}, \mathrm{i}}=\sum_{\mathrm{j}, \mathrm{k}} \mathrm{I}_{\mathrm{cB}, \mathrm{jk}} \Gamma_{\mathrm{ijk}} \\
& \phi_{\mathrm{h}, \mathrm{i}}=\mathrm{z} \sum_{\mathrm{j}, \mathrm{k}} \mathrm{I}_{\mathrm{h}, \mathrm{jk}} \Gamma_{\mathrm{ijk}}
\end{aligned}
$$

in the grand-canonical ensemble.
In terms of our functional expansion, the selfconsistent-field conditions reduce to

$$
\begin{gathered}
\phi_{\mathrm{CA}, \mathrm{i}}-\phi_{\mathrm{CB}, \mathrm{i}}+\phi_{\mathrm{h}, \mathrm{i}}=\left(\mathrm{w}_{\mathrm{B}, \mathrm{i}}-\mathrm{w}_{\mathrm{A}, \mathrm{i}}\right) / \chi \mathrm{N} \\
\phi_{\mathrm{CA}, \mathrm{i}}+\phi_{\mathrm{cB}, \mathrm{i}}+\phi_{\mathrm{h}, \mathrm{i}}=\delta_{1, \mathrm{i}}
\end{gathered}
$$

In the grand-canonical ensemble, these conditions must be satisfied for $\mathrm{i}=1,2,3, \ldots, \mathrm{M}$, but in the canonical ensemble, they only need to be solved for $i=2,3,4, \ldots$, M. This is because $\xi_{1}=(\mathrm{m}+1) \int \rho^{\mathrm{m}} \xi(\rho) \mathrm{d} \rho=0$ in the canonical ensemble, which implies that $\mathrm{w}_{\mathrm{A}, 1}=\chi \mathrm{N} \phi_{\mathrm{CB}, 1}$ $=\chi \mathrm{N}(1-\mathrm{f})(1-\phi)$ and $\mathrm{w}_{\mathrm{B}, 1}=\chi \mathrm{N}\left[\phi_{\mathrm{CA}, 1}+\phi_{\mathrm{h}, 1}\right]=\chi \mathrm{N}[\mathrm{f}(1$ $-\phi)+\phi]$.

Part of our numerical strategy will require that we switch back and forth between the canonical and grandcanonical solutions. To do this, we must be able to evaluate $\mu$ from the canonical solution and likewise extract $\phi$ from the grand-canonical solution. The latter is trivial due to the fact that $\phi=\phi_{\mathrm{h}, 1}$, which is evaluated with eq 43. However, obtaining $\mu$ from the canonical solution is less straightforward. There is also the problem that the fields in the grand-canonical ensemble differ from those of the canonical ensemble by the unspecified constant, $\xi_{1}$. This extra constant transforms the partial partition functions according to $\mathrm{q}_{\mathrm{c}, \mathrm{i}}(\mathrm{s}) \rightarrow \mathrm{q}_{\mathrm{c}, \mathrm{i}}(\mathrm{s})$ $\exp \left(\mathrm{s} \xi_{1}\right), \mathrm{q}_{\mathrm{c}, \mathrm{i}}^{\dagger}(\mathrm{s}) \rightarrow \mathrm{q}_{\mathrm{c}, \mathrm{i}}^{\dagger}(\mathrm{s}) \exp \left((1-\mathrm{s}) \xi_{1}\right)$, and $\mathrm{q}_{\mathrm{h}, \mathrm{i}}(\mathrm{s}) \rightarrow$ $q_{h, i}(s) \exp \left(s \xi_{1}\right)$. Knowing this and requiring the canonical and grand-canonical expressions for the copolymer and homopolymer concentrations to be equivalent leads to the conditions

$$
\xi_{1}=\ln \left(\frac{1-\phi}{q_{c, 1}(1)}\right)=\frac{1}{\alpha} \ln \left(\frac{\phi}{z \alpha q_{h, 1}(\alpha)}\right)
$$

where $q_{c, 1}(1)$ and $q_{h, 1}(\alpha)$ are evaluated in the canonical ensemble. The fact that both equalities must hold implies that the chemical potential is given by

$$
\frac{\mu}{\alpha k_{B} T}=-\ln \left(\frac{1-\phi}{q_{c}(1)}\right)+\frac{1}{\alpha} \ln \left(\frac{\phi}{\alpha q_{h}(\alpha)}\right)
$$

With that, we can now generate the full grand-canonical solution from the canonical one.

C. SCFT for Disordered Phases. In addition to the ordered phases, there are uniform disordered phases in which the fields and concentrations are all constant. In the canonical formalism, their free energy is given by

$$
\begin{aligned}
& \frac{N F_{C}}{\mathrm{k}_{\mathrm{B}} \mathrm{T} \rho_{0} \mathrm{~V}}=(1-\phi)[\ln (1-\phi)-1]+ \\
& \frac{\phi}{\alpha}\left[\ln \left(\frac{\phi}{\alpha}\right)-1\right]+\chi N \phi_{\mathrm{A}}\left(1-\phi_{\mathrm{A}}\right)
\end{aligned}
$$

where $\phi_{\mathrm{A}} \equiv \mathrm{f}(1-\phi)+\phi$ is the total volume fraction of A segments. The grand-canonical free energy can then be evaluated using

$$
\frac{N F_{g c}}{k_{B} T \rho_{0} V}=\frac{N F_{c}}{k_{B} T \rho_{0} V}-\frac{\mu \phi}{\alpha k_{B} T}
$$

where $\phi$ is determined from $\mu$ by solving

$$
\frac{\mu}{\alpha k_{B} T}=-\ln (1-\phi)+\frac{1}{\alpha} \ln \left(\frac{\phi}{\alpha}\right)+\chi N(1-f)\left(1-2 \phi_{A}\right)
$$


There will be some instances of phase coexistence between two uniform disordered phases-one rich in copolymer and the other rich in homopolymer. As $\chi \mathrm{N}$ is lowered, this coexistence will terminate at an I sing-type critical point, where $d^{2} F d d \phi^{2}=d^{3} F d d \phi^{3}=0$. Performing the necessary derivatives in eq 48 locates the critical point at

$$
\begin{aligned}
(\chi N)_{c} & =\frac{(1+\sqrt{\alpha})^{2}}{2 \alpha(1-f)^{2}} \\
\phi_{c} & =\frac{1}{1+\sqrt{\alpha}}
\end{aligned}
$$

D. Disordered-State Scattering Function. The energy penalty of introducing a small sinusoidal profile of wavelength, $2 \pi / \mathrm{k}$, into the disordered phase is inversely proportional to the disordered-state scattering function, $S(k)$, measured by small-angle scattering experiments. It follows that disordered blends become unstable at the first instance where there exists a wavevector, $\mathrm{k}^{*}$, such that $\mathrm{S}^{-1}\left(\mathrm{k}^{*}\right)=0$. The locus of these points defines the disordered-state spinodal curve. Normally, the order-disorder transition (ODT) is predicted to be discontinuous, in which case the spinodal lies slightly inside the ordered region. However, when the transition is to a lamellar phase, the mean-field ODT is predicted to be continuous and consequently coincides with the spinodal. For purposes of calculating these continuous ODT's, we derive S(k) following the SCFT procedure outlined in ref 25, giving us the analytical expression

$$
\mathrm{S}^{-1}(\mathrm{k})=\frac{\phi \mathrm{G}_{\mathrm{h}}+\alpha(1-\phi)\left(\mathrm{S}_{11}+\mathrm{S}_{22}+2 \mathrm{~S}_{12}\right)}{\mathrm{N}(1-\phi)\left[\phi \mathrm{G}_{\mathrm{h}} \mathrm{S}_{22}+\alpha(1-\phi) \operatorname{det}(\mathrm{S})\right]}-2 \chi
$$

where $x \equiv k^{2} a^{2} N / 6, G_{h} \equiv g(\alpha, x), S_{11} \equiv g(f, x), S_{22} \equiv$ $g(1-f, x), S_{12} \equiv h(f, x) h(1-f, x), \operatorname{det}(S) \equiv S_{11} S_{22}-$ $S_{12^{2}}, g(f, x) \equiv 2(\exp (-f x)-1+f x) / x^{2}$, and $h(f, x) \equiv$ $(1-\exp (-\mathrm{fx})) / \mathrm{x}$.

\section{Results}

We now demonstrate our new algorithm on binary blends of $A B$ diblock copolymer and $A$ homopolymer. This is similar to our earlier studies, ${ }^{15,16}$ except that with the UCA we can now explore far higher degrees of segregation al beit without the complex phase behavior. To start, we focus on the effects of changing the diblock composition, $\mathrm{f}$, while holding the homopolymer molecular weight fixed at $\alpha=1.0$. This complements the series of phase diagrams generated by J anert and Schick, ${ }^{17}$ where they varied $\alpha$ at fixed $\mathrm{f}=0.5$.

In mean-field calculations, relevant phase behavior is easily overlooked particularly when working in the grand-canonical ensemble. To avoid this, we routinely plot the canonical free energies, $F_{c}$ of all possible phases over the complete range of homopolymer vol ume fractions, $\phi$, as illustrated in Figure 1 for $\mathrm{f}=0.7$ and $\chi \mathrm{N}=$ 20. It is immediately obvious from this plot that the $L$ phase plays no role in the phase behavior at this particular composition and segregation. Instead, the C phase is favored at low $\phi$, while the $S$ phase becomes stable at high $\phi$. The $\mathrm{C}+\mathrm{S}$ coexistence region could, in principle, be determined by performing a standard double-tangent construction, but instead we use a procedure described in the following section that in-
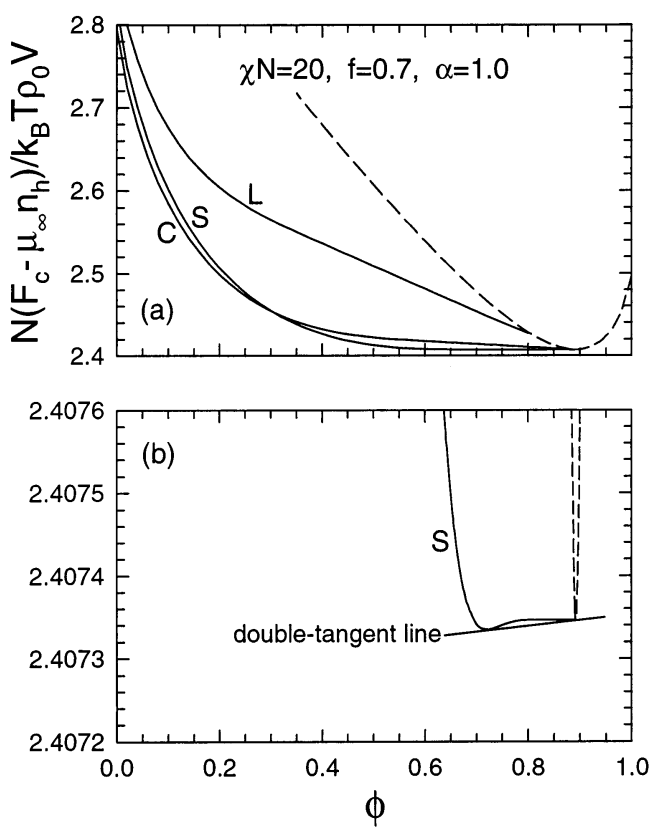

Figure 1. Canonical free energy, $F_{c}$, vs homopolymer vol ume fraction, $\phi$, of the classical phases (solid curves) and the disordered phase (dashed curve) calculated for $\chi \mathrm{N}=20, \mathrm{f}=$ 0.7 , and $\alpha=1.0$. For clarity, the curves have been tilted by plotting $F_{c}-\mu_{\infty} n_{h}$, where $\mu_{\infty}$ is the chemical potential of a highly swollen $S$ phase. Plot b shows an expanded scale in order to highlight the small negative curvature in the free energy of the swollen S phase; the double-tangent construction defines the resulting $S+$ disordered coexistence.

volves a combination of the canonical and grandcanonical ensembles.

As $\phi$ increases, the periodicities of the ordered phases in Figure 1 diverge to infinity, which causes their free energy curves, $F_{c}$ vs $\phi$, to become almost linear and eventually terminate on the dashed curve corresponding to the uniform disordered state. The curves do, however, possess a slight degree of curvature, the sign of which significantly impacts the phase behavior. A positive curvature would result in the continuous unbinding of the ordered phase, ${ }^{26,27}$ while a negative curvature leads to macrophase separation of the homopolymer. Figure $1 \mathrm{~b}$ demonstrates that, in this case, the swollen S phase has negative curvature and shows the double-tangent line defining the $\mathrm{S}+$ disordered region. However, in practice, we locate the coexistence region by yet another method that will also be described in the following section.

By examining these free energy curves over a range of segregations, we construct the phase diagram shown in Figure 2 for diblock copolymers of composition $f=$ $0.7 \mathrm{blended}$ with homopolymers of the same size (i.e., $\alpha$ $=1.0$ ). The pure single-phase regions have been labeled, but the individual two-phase regions (i.e., $\mathrm{C}+\mathrm{S}, \mathrm{S}+$ disordered, and C + disordered) have not. We forego the common practice of separating and labeling the various two-phase regions by including horizontal triple lines (in this case, the $\mathrm{C}+\mathrm{S}+$ disorder line at $\chi \mathrm{N}=30.138$ ) because this would severely clutter some of our diagrams. In any case, it is trivial to identify the coexisting phases by the fact that the tie lines are horizontal. Figure 3 shows an analogous phase diagram, but for a slightly more symmetric diblock of $f=0.6$. Consistent with an increased preference for flatter interfaces, the $L$ phase enters the phase diagram at the expense of the $S$ region. 


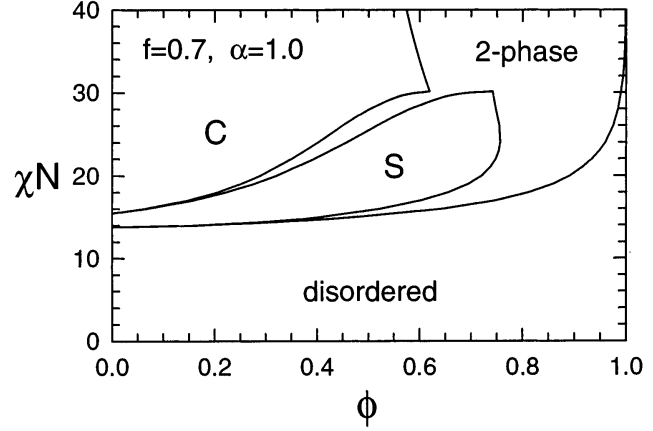

Figure 2. Phase diagram for binary blends containing diblock copolymer of composition $\mathrm{f}=0.7$ and homopolymers of relative molecular weight $\alpha=1.0$ plotted in terms of segregation, $\chi \mathrm{N}$, and homopolymer volume fraction, $\phi$.

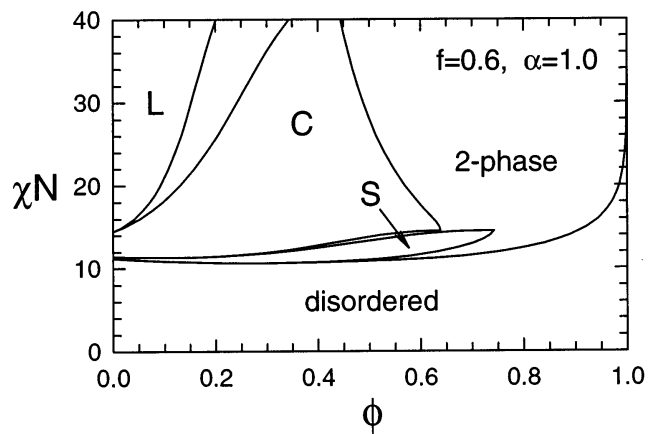

Figure 3. Phase diagram analogous to that in Figure 2, but for diblock copolymers of composition $f=0.6$.

Next, we consider the phase diagram at $\mathrm{f}=0.5$, which has been previously calculated by J anert and Schick.17,26 In that work, they identified some interesting behavior near $\chi \mathrm{N} \approx 11.7$, which has now been called into question. ${ }^{28}$ Therefore, we investigate this segregation more carefully by constructing the free energy curves shown in Figure 4. For clarity, we only show the curves for lamellae (L) and for cylinders with either A-rich cores $\left(C_{A}\right)$ or $B$-rich cores $\left(C_{B}\right)$. Among the ordered phases, $L$ has the lowest energy at all blend compositions. Magnifying its free energy curve to the level of Figure $4 \mathrm{~b}$ reveals a $\mathrm{L}+\mathrm{L}$ coexistence between shortand long-period phases, consistent with the findings of $\mathrm{J}$ anert and Schick. However, further magnification shows that the free energy curve for highly swollen lamel lae has negative curvature, which is inconsistent with the unbinding predicted by J anert and Schick. Instead, the blend favors macrophase separation producing the $L+$ disordered coexistence indicated by the double-tangent line in Figure 4c.

Our revised phase diagram for $f=0.5$ is shown in Figure 5. As originally reported, ${ }^{17}$ this particular composition produces a continuous ODT (dashed curve) that ends at a Lifshitz critical point (solid dot), both of which are calculated from $\mathrm{S}^{-1}(\mathrm{k})$ in eq 53 . The only revision involves the two-phase regions, which are distinguished in Figure 5 by the dotted $\mathrm{L}+\mathrm{L}+$ disordered triple line at $\chi N=11.768$. The small $\mathrm{L}+\mathrm{L}$ region and the large $\mathrm{L}+$ disordered region above the triple line remain the same as in ref 17 , but there is now a second $L+$ disordered region below the triple line that preempts the lamellae from unbinding. The minimum in Figure $4 c$, responsible for this new $L+$ disordered region, becomes too small to resolve when $\chi \mathrm{N} \lesssim 10$, but we have nevertheless extended the phase boundaries down to the Lifshitz point consistent with our belief that the free
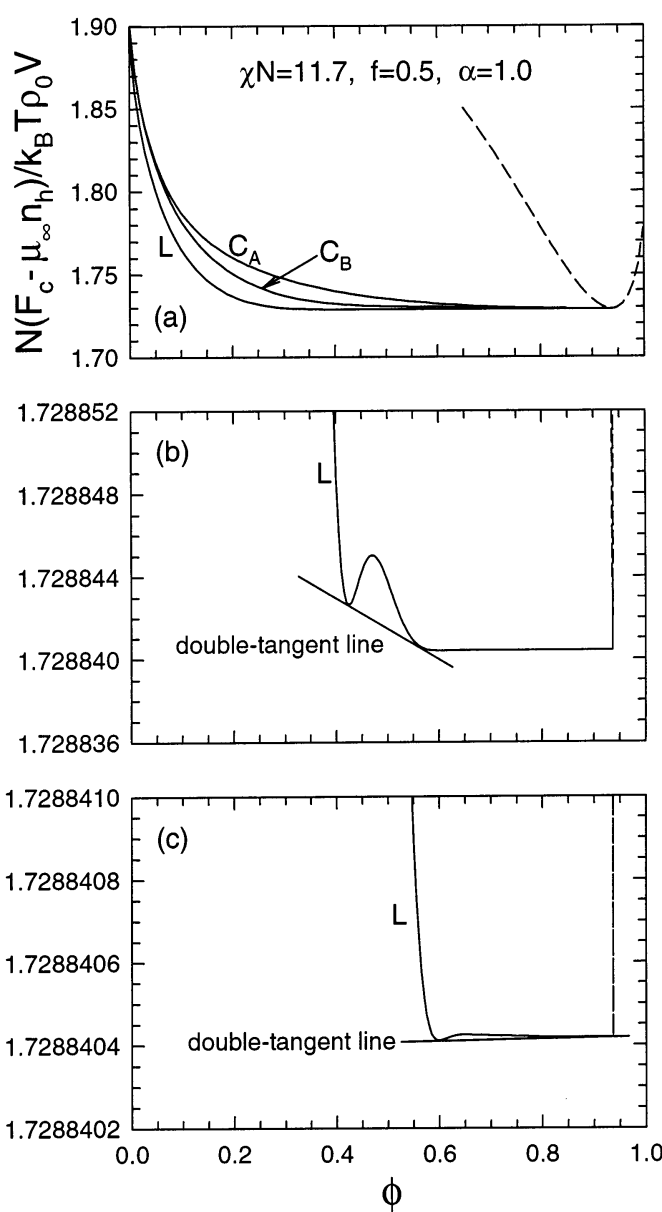

Figure 4. Canonical free energy curves similar to those in Figure 1, but calculated for $\chi \mathrm{N}=11.7, \mathrm{f}=0.5$, and $\alpha=1.0$. The dashed curve corresponds to the disordered phase, while the solid curves correspond to ordered lamellae (L), cylinders with A-rich cores $\left(C_{A}\right)$, and cylinders with B-rich cores $\left(C_{B}\right)$. Plots $b$ and $c$ show increased magnifications so as to highlight the $\mathrm{L}+\mathrm{L}$ and $\mathrm{L}+$ disordered coexistences, respectively.

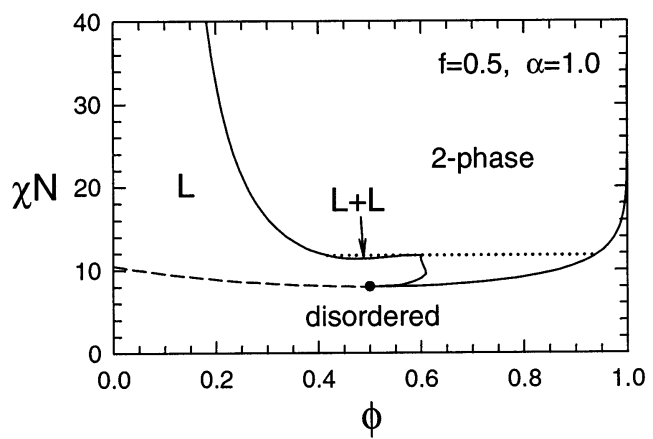

Figure 5. Phase diagram analogous to that in Figure 2, but for diblock copolymers of composition $f=0.5$. The dotted triple line is shown in order to identify the upper boundary of the tiny $L+L$ coexistence region. The dashed curve represents a continuous ODT, and the solid dot denotes a Lifshitz critical point.

energy of highly swollen lamellae is negatively curved at all segregations. Note that Figure 5 has been unaffected by the UCA because it does not involve the $\mathrm{C}$ and $\mathrm{S}$ phases.

For diblock compositions $f<0.5$, the Lifshitz critical point transforms into an Ising-type critical point, above which the blend macrophase separates into two disordered phases-one rich in copolymer and the other rich in homopolymer. Only after the segregation increases 


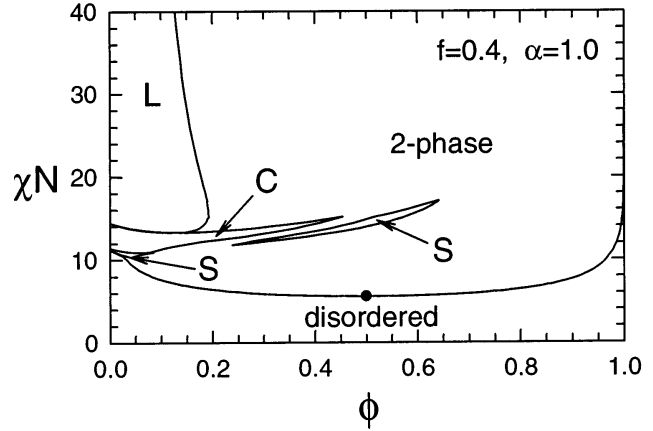

Figure 6. Phase diagram analogous to that in Figure 2, but for diblock copolymers of composition $f=0.4$. The solid dot denotes a critical point above which the blend macrophase separates into two uniform phases-one rich in diblock copolymer and the other rich in homopolymer.

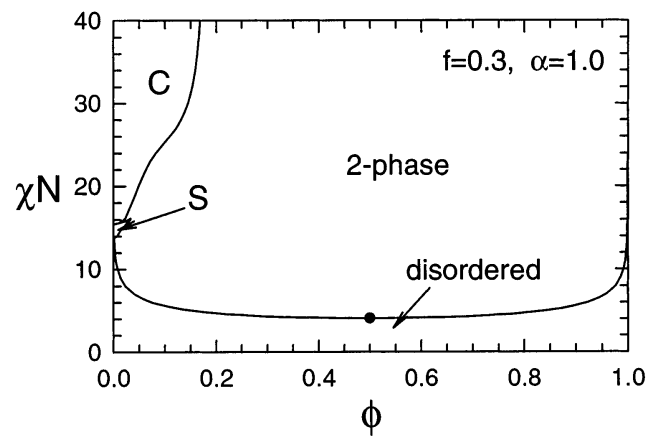

Figure 7. Phase diagram analogous to that in Figure 6, but for diblock copolymers of composition $f=0.3$.

further does the copolymer-rich phase microphase separate into an ordered morphology. This behavior is illustrated in Figure 6 for diblocks with $f=0.4$. Interestingly, the diagram contains highly swollen C and $\mathrm{S}$ regions, similar to those predicted by calculations based on strong-segregation theory (SST). ${ }^{7,10}$ Here the swollen S phase, extending between $0.238<\phi<0.642$, is completely separated from the dry S phase at $\phi<$ 0.076 , whereas the $C$ region is completely connected. Figure 7 shows an analogous phase diagram, but for a diblock asymmetry of $f=0.3$. The swollen phases are no longer present at this composition. Consistent with the fact that the system is becoming an A + B homopolymer blend as $f \rightarrow 0$, the critical point has shifted toward $\chi \mathrm{N} \alpha=2$ and the ordered phases have moved to higher segregation.

The $\chi N-\phi$ phase diagrams examined thus far, analogous to the temperature-composition diagrams typically measured by experiments, ${ }^{29,30}$ are useful for understanding the phase behavior at weak segregations. For stronger segregations, however, the phase boundaries become relatively independent of $\chi \mathrm{N}, 7,10$ and then it becomes more informative to plot phase diagrams in the $\mathrm{f}-\phi$ plane as we have done in Figure 8 for two relatively strong segregations. Indeed, the increase from $\chi \mathrm{N}=20$ to 40 has relatively little effect on these diagrams other than to spread the phase boundaries apart al ong the $f$ axis. This simple trend accounts for the $\mathrm{L} \rightarrow \mathrm{C} \rightarrow \mathrm{S}$ progression observed in Figures 2 and 3 with increasing segregation. The same general progression that occurs in Figures 2 and 3 for increasing homopolymer content is attributed instead to the tilt in the order-order phase boundaries on the $f \approx 0.5$ side of the $\mathrm{f}-\phi$ phase diagrams. Figure 8 also shows, far more clearly than the previous $\chi \mathrm{N}-\phi$ diagrams, the
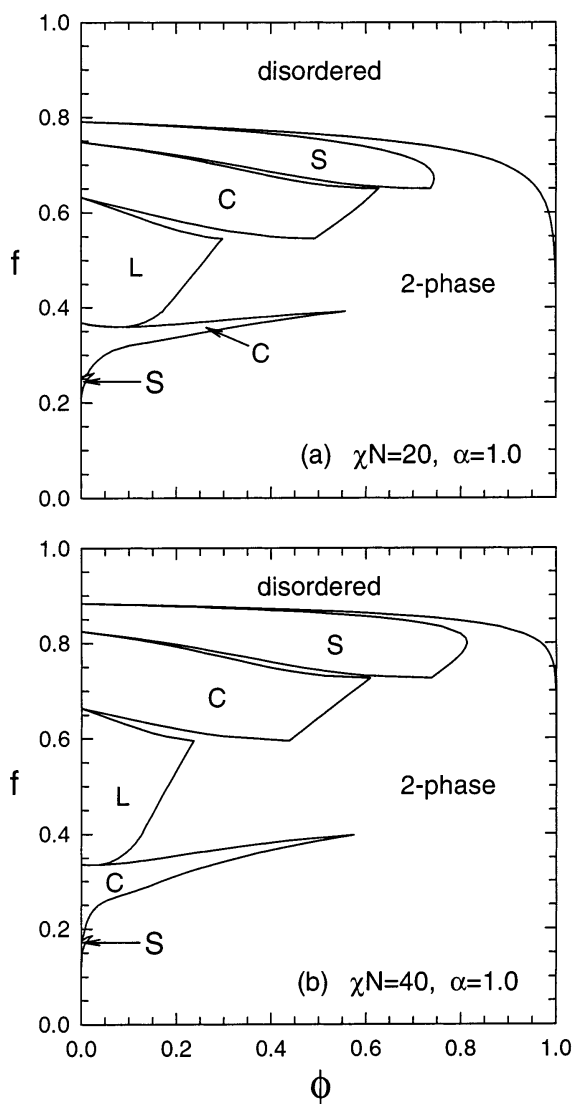

Figure 8. Phase diagrams in terms of diblock composition, $f$, and homopolymer volume fraction, $\phi$, calculated for segregations of (a) $\chi \mathrm{N}=20$ and (b) $\chi \mathrm{N}=40$.

regions where $\mathrm{C}$ and $\mathrm{S}$ are swollen by minority-type homopolymer (at $f \lesssim 0.5$ ). In particular, it shows that the degree of swelling increases rapidly as the diblock copolymer becomes more symmetric. Note that this swelling by minority homopolymer is in much better qualitative agreement with the SST predictions of ref 10 than the earlier and more approximate ones of ref 7.

The swelling of a microstructure by majority-type homopolymer $(f \geq 0.5)$ is distinctly different from swelling by minority-type homopolymer $(f \lesssim 0.5)$. The segment profiles, $\phi_{\mathrm{CA}}(r), \phi_{\mathrm{CB}}(r)$, and $\phi_{\mathrm{h}}(r)$, for oppositely swollen $C$ phases are shown in Figure 9 for conditions corresponding to $\mathrm{L}+\mathrm{C}+$ disordered coexistence. In plot a where the copolymer cylinders have B-rich cores, the homopolymer swells the matrix pushing the cylinders apart. Here the homopolymer creates an effective interaction between the cylindrical units. ${ }^{31}$ At short distances, the interaction between cylinders is repulsive due to the translational entropy the homopolymer gains by mixing with their A-rich exteriors, and this favors swelling. However, at large distances, the configurational entropy the homopolymer loses by having to avoid the copolymer cylinders becomes increasingly important, causing the effective interaction to become attractive. As soon as this happens, the swelling stops and the excess homopolymer macrophase separates. ${ }^{28}$ In plot $\mathrm{b}$, the homopolymer swells the interior of the cylindrical units. Since the homopolymer region is essentially pure, it is impossible for it to mediate an effective interaction between the copolymer layers, and thus the behavior must instead be governed by the elastic properties of the copolymer layers. ${ }^{31}$ By taking on homopolymer, the copolymer layers are able to reduce their mean curva- 

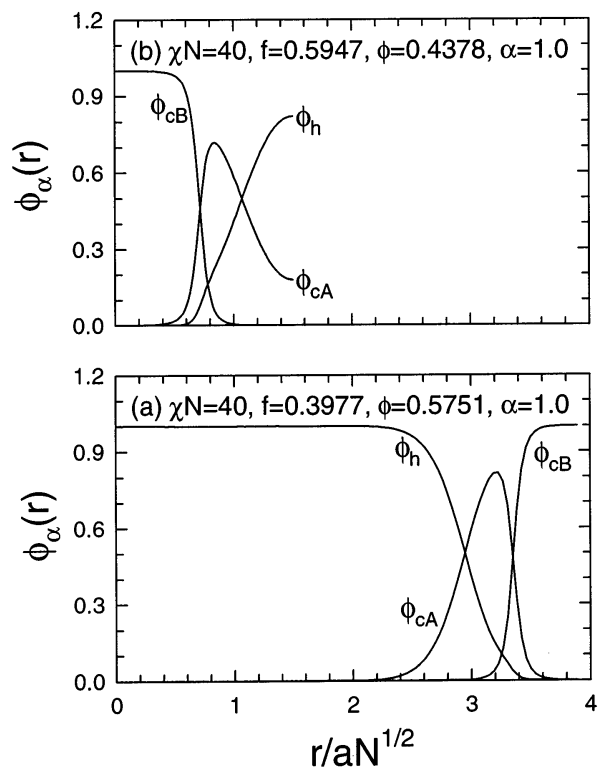

Figure 9. Segment profiles of the copolymer A segments, $\phi_{\mathrm{CA}}(r)$, the copol ymer B segments, $\phi_{\mathrm{CB}}(r)$, and the homopolymer, $\phi_{\mathrm{h}}(\mathrm{r})$, for swollen cylinder (C) phases calculated at $\mathrm{L}+\mathrm{C}+$ disordered coexistence. Plot a shows the $C$ phase from the $f>$ 0.5 side of the phase diagram where the cylinder cores are B-rich, and plot $b$ shows it from the $f<0.5$ side where the cores are A-rich.

ture, $H=1 / 2 R_{A B}$, where $R_{A B}$ is the radius of the $A B$ interface generally defined by $\phi_{C A}\left(R_{A B}\right)+\phi_{h}\left(R_{A B}\right)=$ $\phi_{C B}\left(R_{A B}\right)=0.5$. The swelling presumably continues up until $\mathrm{H}$ is reduced to the preferred or spontaneous curvature, $\mathrm{H}_{0}{ }^{31}$ The fact ${ }^{4}$ that $\mathrm{H}_{0} \rightarrow 0$ as $\mathrm{f} \rightarrow 0.5$ accounts for the extra swelling by minority homopolymer observed in Figure 8 as the diblock copolymer becomes increasingly symmetric.

The theoretical study by J anert and Schick ${ }^{17}$ has al ready demonstrated for the special case of symmetric diblocks (i.e., $f=0.5$ ) that the homopolymer molecular weight (i.e., $\alpha$ ) has a substantial impact on the phase diagram. Although it is beyond the scope of this paper to extend their thorough examination to the full range of asymmetric diblocks, Figure 10 does illustrate its importance by showing $\mathrm{f}-\phi$ phase diagrams for slightly larger homopolymers where $\alpha=1.5$. Figure 11 also shows how the segment profiles of the swollen $C$ phases are affected. Both figures demonstrate a diminished tendency for larger homopolymers to mix with the copolymer microstructures. This is linked to a reduction in homopolymer translational entropy, which is proportional to the number of homopolymer molecules, $n_{h} \equiv$

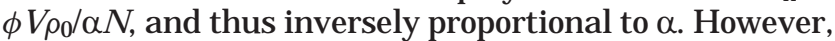
the exact mechanism does differ between the two sides of the phase diagram. On the $\approx 0.5$ side, the reduction in translational entropy causes the attraction between the copolymer layers to occur at shorter range. ${ }^{31}$ On the $\mathrm{f} \lesssim 0.5$ side, the drop in translational entropy reduces the penetration of homopolymer into the A-rich portion of the copolymer structures, causing $\mathrm{H}_{0}$ to increase; the penetration normally stretches the A blocks, creating a more even balance with the stretching energy of the longer B blocks, resulting in preference for flatter copolymer layers. According to SST calculations for the limit of $\alpha \rightarrow \infty, 7,10$ large majority-type homopolymers become virtually incompatible with the copolymer microstructures, but the swelling by large minority-type homopolymers remains significant.
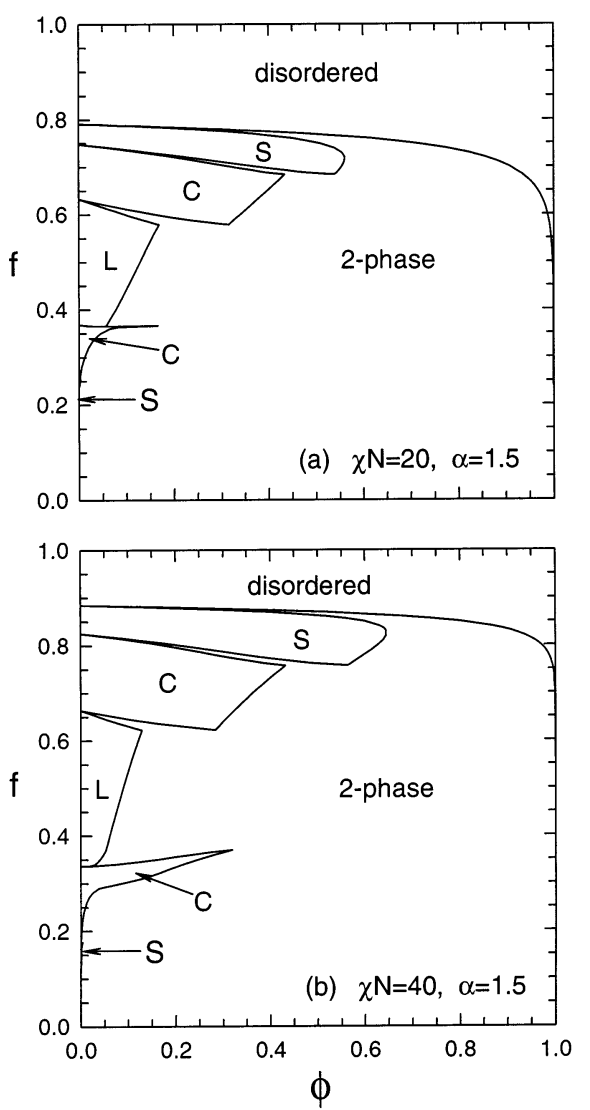

Figure 10. Phase diagrams analogous to those in Figure 8, but for larger homopolymer molecules with $\alpha=1.5$.
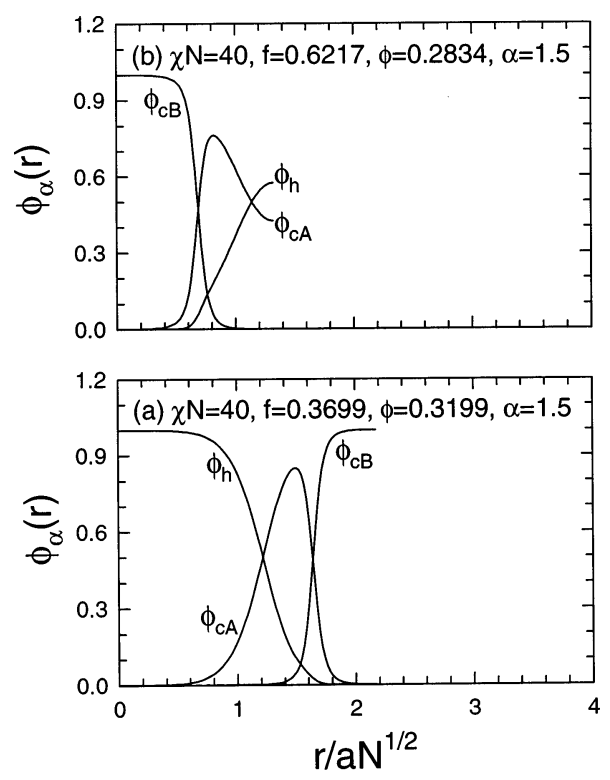

Figure 11. Segment profiles analogous to those in Figure 9, but for larger homopolymer molecules with $\alpha=1.5$. For comparison purposes, the profiles are plotted on the same length scale used in Figure 9.

\section{Discussion}

The utility of our new SCFT algorithm has been successfully demonstrated by its application to binary diblock copolymer/homopolymer blends. Key to its superb performance is the unit-cell approximation (UCA), but this implies an insensitivity to the actual packing arrangement of, for example, the spheres (e.g., bcc or $\left.f_{c c}\right)^{29,32}$ and excludes the treatment of complex morphol- 
ogies (e.g., gyroid, double-diamond, and perforatedlamellar). ${ }^{33}$ Nevertheless, the packing arrangement is just fine detail that could potentially be deduced by examining the effective interaction between polymeric brushes. ${ }^{34}$ Even the omission of complex morphologies is not a serious limitation for numerous systems where the complex phases only enter in narrow channels between the $L$ and $C$ phases. In fact, this appears to be the case for virtually all block copolymer architectures composed of two chemically distinct segments (i.e., A and $B$ ) and even remains so when they are mixed with solvent. ${ }^{28,29}$ However, the addition of a third chemical species to the copolymer, as in ABC triblocks, ${ }^{1}$ generally results in a phase diagram dominated by nonclassical morphologies, in which case the UCA becomes inappropriate.

Previous comparisons ${ }^{20}$ with full SCFT calculations have established that the UCA is reasonably accurate for neat diblock copolymer blends. We have similarly tested a selection of points on the phase boundaries in Figure $8 \mathrm{a}$ for $\chi \mathrm{N}=20$ and $\alpha=1.0$. Here, the addition of homopolymer does not diminish the accuracy of the UCA, except in regards to the narrow channel of highly swollen cylinders at $f \approx 0.35$; the extension of $C$ into the two-phase region is reduced by about one-third when the UCA is removed. The explanation is straightforward; on this side of the phase diagram the copolymer boarders the edge of the unit cell and is thus significantly affected by the shape of the unit cell. More specifically, the hexagonal unit cell prevents the copolymer layer from acquiring a uniform curvature, which reduces its stability. Packing frustration ${ }^{18}$ effects such as this will be discussed more thoroughly in a forthcoming paper that will also address the complex phase behavior.

The UCA has been used in numerous other SCFT calculations, ${ }^{2,19}$ but they solved the self-consistent-field equations in real space rather than with a functional expansion. 22 For UCA-based calculations, our spectral method offers a number of distinct advantages, not least of which is the ability to evaluate integrals over s and $\rho$ analytically. In addition to the improved speed, this reduces the sources of numerical uncertainty. In the real-space approach, there are three diffusion equations and numerous integrations that have to be solved on grids, each with a finite step size that contributes to the numerical inaccuracy. The accuracy is further reduced by their use of a Picard iteration scheme ${ }^{35}$ to solve the self-consistent-field equations. In contrast, the spectral method has only one significant source of numerical uncertainty to monitor, that due to the truncation of the functional expansion. The algorithm for diagonalizing the matrices is so exceptionally accurate that its contribution to the numerical error is inconsequential. Although we do occasionally use the Picard iteration scheme because of its superior stability, we always obtain our final solution for the selfconsistent fields with the Boyden algorithm. ${ }^{24}$ With its rapid exponential-like convergence, we can be extremely strict with the error tolerance to the point that the numerical uncertainty is absolutely irrel evant. ${ }^{36} \mathrm{Fur}$ thermore, the Boyden method allows us to minimize the free energy with respect to $R$ at the same time it solves the field equations, providing a significant saving in computational time.

The computational speed of our algorithm is highly sensitive to the number of basis functions, $M$, retained in the calculation. For $M=20$, it takes a mere $0.1 \mathrm{~s}$ (using a Compaq $667 \mathrm{MHz}$ Alpha 21264 processor) to obtain the complete solution of an ordered phase. This increases to $1 \mathrm{~s}$ for $\mathrm{M}=40$ and to $10 \mathrm{~s}$ for $\mathrm{M}=80$. F or large $\mathrm{M}$, the computational time scal es as $\mathrm{M}^{4}$, of which three powers are attributed to the matrix diagonalization and one power is from calculating a $J$ acobian used by the Boyden method. With the UCA, M = 20 is generally sufficient to obtain 9 significant figures of accuracy in the free energy, but without the UCA, this number escalates to about $M=1000$. Given the $M^{4}$ scaling, it is easy to understand the tremendous computational advantage provided by the UCA.

The combined use of the canonical and grand-canonical ensembles has a number of distinct advantages. The canonical algorithm is more stable because the homopolymer vol ume fraction is fixed, but the grand-canonical ensemble avoids the need to perform double-tangent constructions. In many instances, especially if the coexistence region is narrow, as in Figure 1a, it is best to first locate the homopolymer volume fraction, $\phi$, where the two phases have the same $F_{c}$, using the canonical algorithm. Naturally, the solutions will not be in equilibrium because they have different chemical potentials, but they are usually close to equilibrium. Consequently, the average of their chemical potentials and their solutions for $\mathrm{w}_{\mathrm{A}, \mathrm{i}}, \mathrm{W}_{\mathrm{B}, \mathrm{i}}$, and $\mathrm{R}$ provides an ideal initial guess for the less stable grand-canonical algorithm, which obtains the coexisting solutions by simply equating the corresponding free energies, $F_{\text {gc. }}$ The coexistence region between ordered and disordered phases, as in Figure 1b, can be particularly difficult to locate, and for that we have another procedure. It starts with a guess for the blend composition, $\phi$, of the ordered phase, for which $F_{c}$ is calculated using the canonical algorithm. Next, $\mu$ is calculated using eq 47, allowing us to evaluate $\mathrm{F}_{\mathrm{gc}}=\mathrm{F}_{\mathrm{c}}-\mu \mathrm{n}_{\mathrm{h}}$. Then, $\mathrm{F}_{\mathrm{gc}}$ is calculated for the disordered phase at the same $\mu$, following the procedure described in section 2.3. Finally, $\phi$ is adjusted according to the Newton-Raphson procedure ${ }^{24}$ until the ordered and disordered phases have the same $F_{\text {gc. }}$

The phase behavior of block copolymer blends represents a far greater challenge than that of neat block copolymer melts, largely because of the possibility of phase coexistence. With this added complexity comes a greater potential for mistaken predictions, and this is why we strongly advocate the use of $F_{c}$ vs $\phi$ plots like those in Figures 1 and 4 . Certainly our correction to the $\mathrm{f}=0.5$ phase diagram in Figure 5 , regarding macrophase separation vs unbinding of lamellae, was greatly aided by the use of Figure 4 . Similar corrections ${ }^{28,37}$ have also been made for ternary blends of diblock copolymer with both parent homopolymers. In yet another documented case, ${ }^{28}$ ref 27 incorrectly predicted a coexistence between asymmetric lamellar phases in symmetric ternary blends, which definitely would not have happened had they examined $F_{c}$ vs $\phi$.

The $L+L$ coexistence in Figure 5 , where there exist two equally preferred lamellar thicknesses, has also been predicted for ternary diblock copolymer/homopolymer blends. In that case, a detailed examination ${ }^{28}$ of the effective interaction between copolymer layers concluded that, to an exceptional accuracy, the system is insensitive to the actual sequence of thin and thick lamellae. Given this, the thin and thick lamellae are bound to form an irregular sequence, resulting in a unique nonperiodic lamellar phase. Binary blends are 
likely to behave the same, but to be certain would require a detailed investigation of the effective interaction between diblock bilayers, which is beyond the scope of this paper.

Naturally, our mean-field predictions will be modified somewhat by fluctuations, particularly for low-molecular-weight polymers. These effects, which are controlled by the invariant polymerization index, $\overline{\mathrm{N}} \equiv \rho_{0}{ }^{2} \mathrm{a}^{6} \mathrm{~N}$, become less important for larger polymer molecules. The weakly segregated morphologies are most prone to fluctuations, and hence the changes to the phase diagram are most significant at small $\chi \mathrm{N}$. For example, the continuous ODT in Figure 5 will become discontinuous and the Lifshitz critical point will be destroyed. As discussed before, ${ }^{16}$ strongly segregated morphologies swollen by majority-type homopolymer will also be disrupted by fluctuations, transforming them into disordered micellar regions once the effective interaction between the copolymer units (e.g., cylinders or spheres) becomes too weak to maintain their long-range order. Another more intriguing possibility is that the lamellar phase above the Lifshitz critical point in Figure 5 might transform into a sponge morphol ogy ${ }^{40}$ analogous to the bicontinuous mi croemulsion observed in ternary blends. ${ }^{41}$ The sponge region would presumably exist in a vertical channel connected to the disordered phase and extending upward, possibly consuming the $\mathrm{L}+\mathrm{L}$ region.

Although fluctuation effects have not yet been studied in binary blends, they have been investigated in ternary blends containing both parent homopolymers. Kiel horn and Muthukumar ${ }^{42}$ examined them by extending the standard approach developed by Fredrickson and $\mathrm{Hel}$ fand ${ }^{38}$ for neat diblock copolymer melts. The fact that these calculations are based on a mean-field Landau free energy functional does, however, raise questions regarding their reliability, ${ }^{4}$ but more concerning is a recent calculation by Kudlay and Stepanow ${ }^{43}$ showing that the $\mathrm{F}-\mathrm{H}$ approach breaks down as certain approximations are removed. Müller and Schick ${ }^{13}$ have performed Monte Carlo simulations, which are undoubtedly more reliable, but they were restricted to low $\overline{\mathrm{N}} \approx$ 100 , which is much smaller than the values, $\overline{\mathrm{N}} \sim 10^{3}$ $10^{4}$, typical of experiment. ${ }^{44}$ Field-theoretic simulations ${ }^{39,37}$ may eventually provide reliable fluctuation predictions for larger $\overline{\mathrm{N}}$, but for now their high computational demands have restricted their application to two-dimensional space.

Given that the weakly segregated morphologies (i.e., $\chi \mathrm{N} \lesssim 15$ ) in the mean-field phase diagram are destroyed by fluctuations, SCFT calculations performed in this regime will only be meaningful provided that they are qualitatively similar to those at Iarger $\chi \mathrm{N}$. Since our $\chi \mathrm{N}-\phi$ phase diagrams, including some not presented here, indicate some qualitative differences, appropriate caution should, therefore, be taken when considering our earlier SCFT predictions. ${ }^{15,16}$ Still, they should be far more reliable than those ${ }^{11}$ based on weak-segregation theory (WST), which generally assumes a singleharmonic approximation with small-amplitude sinusoidal profiles.4,9,45 A serious pitfall of the singleharmonic approximation has already been demonstrated. ${ }^{46}$ In any case, there is no longer any need to concern ourselves with such issues now that SCFT calculations can easily be performed at segregations well beyond the fluctuation regime. This ability is particularly valuable considering the limitations of strongsegregation theory (SST). ${ }^{4,8,9}$ Although successful for neat diblock copolymer melts, SST becomes problematic when applied to blends. On the basis of comparisons with SCFT, 34 SST is unable to accurately treat either the effective interaction between block copolymer layers or the tendency of homopolymer to penetrate into them, both of which are central to the behavior of block copolymer/homopolymer blends.

\section{Summary}

Self-consistent-field theory (SCFT) offers the most complete and accurate treatment of block copolymer systems, ${ }^{4,9}$ next to actual Monte Carlo and molecular dynamics simulations. ${ }^{12-14,37,38}$ It provides exact meanfield predictions for the Gaussian coarse-grained microscopic model, ${ }^{4}$ which has become the standard among block copolymer theories. Unfortunately, the numerical demands of SCFT have deterred researchers toward less accurate theories.

By supplementing SCFT with the common unit-cell approximation (UCA $)^{2,19}$ and then solving the relevant equations with a functional expansion, ${ }^{22}$ we have drastically reduced the computational demands of SCFT. The time required for a single-processor computer to evaluate the complete solution of a well-segregated morphology is now generally less than $1 \mathrm{~s}$. The only restriction is that the method is limited to the classical lamellar, cylindrical, and spherical morphologies, but there are many block copolymer systems for which this is not a serious drawback.

To demonstrate the method, we have examined binary blends of $A B$ diblock copolymer and A homopolymer, focusing on the case where the two molecules are of equal size. A full complement of phase diagrams has been calculated up to $\chi \mathrm{N}=40$, which is considerably higher than in previous SCFT studies, ${ }^{15-17}$ but still nowhere near the practical limitations of our method. The method is equally amenable to more complex block copolymer architectures, ${ }^{47}$ and can easily be extended to any number of molecular species. At the moment, this new SCFT-based algorithm seems to offer the only reliable way for researchers to navigate the vast parameter spaces involved in many block copolymer blends.

Acknowledgment. This work was supported by EPSRC (GR/N 36721) and the Dow Chemical Co.

\section{References and Notes}

(1) Bates, F. S.; Fredrickson, G. H. Phys. Today 1999, 52, 32.

(2) Helfand, E.; Wasserman, Z. R. Macromol ecules 1978, 11, 960; Macromol ecules 1980, 13, 994.

(3) Helfand, E. J . Chem. Phys. 1975, 62, 999.

(4) Matsen, M. W. J . Phys.: Condens. Matter 2002, 14, R21.

(5) Matsen, M. W. J . Chem. Phys. 1998, 108, 785. Shefel bine, T. A.; Vigild, M. E.; Matsen, M. W.; Hajduk, D. A.; Hillmyer, M. A.; Bates, F. S. J . Am. Chem. Soc. 1999, 121, 8457.

(6) Matsen, M. W. J . Chem. Phys. 1995, 103, 3268. Papadakis, C. M.; Mortensen, K.; Posselt, D. Eur. Phys. J . B 1998, 4, 325.

(7) Semenov, A. N. Macromolecules 1993, 26, 2273

(8) Semenov, A. N. Sov. Phys. J ETP 1985, 61, 733.

(9) Matsen, M. W.; Bates, F. S. Macromolecules 1996, 29, 1091.

(10) Likhtman, A. E.; Semenov, A. N. Macromolecules 1994, 27, 3103.

(11) Huh, J .; J o, W. H. J . Chem. Phys. 2002, 117, 9920.

(12) Vassiliev, O. N.; Matsen, M. W. J . Chem. Phys. 2003, 118, 7700.

(13) Müller, M.; Schick, M. J . Chem. Phys. 1996, 105, 8885.

(14) Murat, M.; Grest, G. S.; Kremer, K. Macromol ecules 1999, 32, 595.

(15) Matsen, M. W. Phys. Rev. Lett. 1995, 674, 4225. 
(16) Matsen, M. W. Macromolecules 1995, 28, 5765.

(17) J anert, P. K.; Schick, M. Macromol ecules 1998, 31, 1109.

(18) Matsen, M. W.; Bates, F. S. Macromolecules 1996, $29,7641$.

(19) Vavasour, J . D.; Whitmore, M. D. Macromol ecules 1992, 25 2041; Macromolecules 1992, 25, 5477. Shi, A.-C.: Noolandi, J . Macromol ecules 1994, 27, 2936; Macromol ecules 1995, 28, 3103.

(20) Matsen, M. W.; Whitmore, M. D. J . Chem. Phys. 1996, 105, 9698.

(21) Hong, K. M.; Noolandi, J . Macromolecules 1981, 14, 727.

(22) Matsen, M. W.; Schick, M. Phys. Rev. Lett. 1994, 72, 2660.

(23) Gradshteyyn, I. S.; Ryzhik, I. M. Table of Integrals, Series, and Products; Academic Press: New York, 1980; p 981.

(24) Burden, R. L.; Faires, J. D.; Reynolds, A. C. Numerical Analysis, 2nd ed.; PWS Publishers: Boston, 1981.

(25) Naughton, J. R.; Matsen, M. W. Macromol ecules 2002, 35, 5688; note that the three plus signs in eq 40 should all be minus signs.

(26) J anert, P. K.; Schick, M. Phys. Rev. E 1996, 54, R33.

(27) J anert, P. K.; Schick, M. Macromolecules 1997, 30, 3916.

(28) Naughton, J. R.; Matsen, M. W. Macromolecules 2002, 35, 8926.

(29) Lodge, T. P.; Pudil, B.; Hanley, K. J . Macromolecules 2002 35, 4707. Hanley, K. J .; Lodge, T. P.; Huang, C. I. Macromolecules 2000, 33, 5918.

(30) Lee, S. H.; Char, K.; Kim, G. Macromolecules 2000, 33, 7072.

(31) Thompson, R. B.; Matsen, M. W. J . Chem. Phys. 2000, 112, 6863.

(32) Huang, Y.-Y.; Chen, H.-L.; Hashimoto, T. Macromolecules 2003, 36, 764 .

(33) Zin, W.-C.; Ahn, A.-H. Macromolecules 2002, 35, 10238.

(34) Matsen, M. W.; Gardiner, J . M. J . Chem. Phys. 2001, 115, 2794.
(35) Shull, K. R. Macromolecules 1993, 26, 2346. Laradji, M.; Shi, A.-C.; Noolandi, J .; Desai, R. C. Macromolecules 1996, 29, 6487.

(36) We calculate the J acobian using finite differences, but this does not affect the numerical accuracy of the resulting solution for the self-consistent fields. All it does is slight reduce the rate of convergence.

(37) Düchs, D.; Ganesan, V.; Fredrickson, G. H.; Schmid, F. Macromolecules 2003, 36, in press.

(38) Fredrickson, G. H.; Helfand, E. J . Chem. Phys. 1987, 87, 697

(39) Fredrickson, G. H.; Ganesan, V.; Drolet, F. Macromolecules 2002, 35, 16.

(40) Roux, D.; Coulon, C.; Cates, M. E. J . Phys. Chem. 1992, 96, 4174. Hecht, E.; Mortensen, K.; Hoffmann, H. Macromolecules 1995, 28, 5465.

(41) Bates, F. S.; Maurer, W.; Lipic, M. P.; Hillmyer, M. A.; Almdal, K.; Mortensen, K.; Fredrickson, G. H.; Lodge, T. P. Phys. Rev. Lett. 1997, 79, 849.

(42) Kielhorn, L.; Muthukumar, M. J . Chem. Phys. 1997, 107, 5588.

(43) Kudlay, A.; Stepanow, S. J . Chem. Phys. 2003, 118, 4272

(44) Bates, F. S.; Schulz, M. F.; Khandpur, A. K.; Förster, S.; Rosedale, J. H.; Almdal, K.; Mortensen, K. Faraday Discuss. 1994, 98, 7 .

(45) Leibler, L. Macromol ecules 1980, 13, 1602.

(46) Fredrickson, G. H.; Leibler, L. Macromolecules 1989, 22, 1238.

(47) Matsen, M. W.; Schick, M. Macromolecules 1994, 27, 6761; Macromolecules 1994, 27, 7157.

MA0349377 